\section{CLINICAL LECTURES ON}

\author{
N E R V O U S D I S E A S E S.
}

By A. HUGHES BENNETT, M.D., M.R.C.P., Physician to the Hospital for Epilepsy and Paralysis, Regent's Park; Assistant Physician to the Westminster Hospital ; etc.

LECTURE III (continued from page 48).

Electricity as a Method of Physical Diagnosis in Diseases OF THE NeRvous SySTEM.

II. The Actions of Faradism and Galvanism upon Injured and Diseased Living Tissues. - We have seen that in healthy living tissues electricity produces definite and almost constant effects; that its general action, in moderate doses, is to stimulate into action the different functions peculiar to each; and this it does in a manner proportionate to the functional activity of the structure and to the intensity of the current. When such tissues are injured or diseased, and their function consequently augmented, diminished, or annihilated, the electrical reactıons are modified in a corresponding manner. It is by a study of the anatomical and histological changes in health and disease, as compared to their varied reactions to faradism and galvanism under different circumstances, that we are enabled to arrive at valuable information in diagnosis and prognosis. It may be said, in general terms, that, if an organ or structure be diseased, its function will be altered, and that electricity will fail to induce the normal reactions; if there be only partial disorganisation, function may to some extent remain, accompanied by modified electrical changes; but if there be complete destruction, all electrical, as well as all functional, activity will be abolished. Our knowledge of the whole subject is still in its infancy; but we shall endeavour to describe in detail the reactions of electricity, as far as we know them, in various abnormal conditions of the nervous system.

The Brain. - If any portion of the brain be injured or diseased, its function will be proportionately destroyed and its response to electricity lost. If, for example, the centre on the surface of the cerebral convolution which regulates the movements of certain muscles be completely diseased, the voluntary actions of these parts will be absent, and direct stimulation by electricity of their nucleus will fail to produce the natural contractions. Also, if any portions of the motor or sensory tracts be injured, electricity will be unable to convey to and from the centres its natural influences. In paralysis from brain-disease, it is found that, as a rule, the electrical reactions of the spinal cord, nerve, and muscle, remain normal. If the injury or disease produce local irritation, they are sometimes exaggerated, and these structures respond to a more feeble stimulus than ordinary, showing them to be more sensitive than natural. Another explanation of this increased electrical action in such cases is this: owing to the lesion, the inhibitory influences of the brain upon the spinal cord and nerves are interrupted, and thus as a result of external stimulus, motor activity is augmented below the seat of the disease. In very chronic cases of brain-paralysis, when the limbs have been long out of use, there may be slight diminution of electrical excitability; and in very advanced instances, secondary anatomical changes may take place, which would be accompanied by modification in the irritability of the cord, nerve, and muscle. These we shall subsequently discuss when treating of each in turn. It may, however, be again stated that, in paralysis from cerebral disease, the general rule is for the electrical conditions to be normal, and this, as we shall afterwards see, is a fact of great diagnostic value. Typical examples of these negative reactions may be seen in hæmorrhage, softening, tumour, etc.

Spinal Cord.-Experiments on animals have shown that, if the spinal cord be divided at any point, there is paralysis below the section; and if electricity be applied to the upper portion, there are contractions of all the muscles supplied from that segment, but those receiving their influence from the lower part remain unaffected. The same takes place if the inferior segment be irritated, only then the lower limbs are convulsed and the upper portion of the body is not influenced. The same phenomena ensue, although the divided edges be brought into contact, showing that the functional activity of a portion of the cord has been interfered with by the section, as when the ends are joined there is no interference with the conductive power of the nerve-structures, which remain as before. In such a case, the paralysed limbs would not undergo any disorganisation, and their nerves and muscles would give normal or increased electrical reactions. If a portion of the whole thickness of the cord were destroyed, say, for an inch or two in length, above the lum bar region, there would be somewhat different reactions. The sym- ptoms would be loss of motion in the lower part of the body. After some time, muscular wasting and loss of reflex action would ensue in that portion of the paralysed part supplied from the diseased segment of cord, but no wasting or loss of reflex action in the lower limbs. The electrical conditions would be as follows. If electricity were applied to the part of the cord diseased, no reaction of any kind would ensue. The nerves and muscles derived from theinjured portion would exhibit the characteristic changes of nerve and muscle degeneration, which will subsequently be described. Electricity applied to the healthy portion of the cord below the disease would cause natural or increased contractions in the lower and paralysed limbs, and these would also take place when the nerves and muscles were similarly treated. If the whole spinal cord were artificially destroyed, there would be loss of motion and of reflex action throughout the trunk and limbs. If the animal lived, there would subsequently be wasting of the nerves and muscles. Electrical irritation of the cord itself would induce no contractions, and there would be the reaction of general muscular and nervous degeneration. These phenomena indicate that, if the cord be injured at the origin of a motor nerve, the latter rapidly loses its healthy structure and functional activity, its nutrition is cut off, it degenerates, and with it the muscle it supplies; both atrophy, and both lose electrical as well as voluntary excitability. They also indicate that so long as a motor nerve is attached to a healthy centre, such as the lower portion of the cord, even although it may be separated from the brain, and consequently voluntary motion lost, the nerves and muscles retain their electric excitability.

In man, the same general sequence of events ensues after injury or disease of the spinal cord, although, owing to the nature of the lesion, we have rarely opportunities of observing such typical symptoms as in experiments on the lower animals. When a thin section of the cord is injured, as when divided by a dagger, the voluntary motor power is lost, and reflex action, if affected, is increased. The electrical reactions are normal to the parts supplied from the upper segment, and are increased to those deriving their influence from the lower portion. There is no degeneration of the cord, nerve, or muscle. When the injury affects the whole thickness of the cord for some extent, as from the pressure of a fractured vertebra, the symptoms and reactions are at first the same as those just mentioned, except that reflex action is arrested in those nerves deriving their influence from the injured portion. After a time, degenerative changes not only ensue in the diseased portion of the cord, but would extend to the nerves and muscles supplied from it. When fully developed, the sym. ptoms and electrical actions are loss of voluntary power from and below the injured part, loss of reflex action to the upper parts of the paralysed portion, with degeneration of nerve and atrophy of muscle. In the parts supplied from that segment of the cord below the diseased portion, there is loss of voluntary power, but reflex action is retained, and there is no degeneration of nerve or wasting of muscle, as, when attached to healthy centres, these long retain their bulk and irritability. In the first instance, there are the typical electrical reactions of degenerated nerve and muscle; in the latter, the electric excitability is either not affected or is increased.

In disease, the same physical conditions of the cord may exist. In so-called myelitis transversa, when a portion of the whole thickness of the cord is diseased, the symptoms and electrical reactions are the same as those just described. If the anterior or antero-lateral columns of the cord only be diseased, as in lateral sclerosis, there is motor paralysis, no loss of reflex action, and no atrophy of nerve or muscle. The electrical reactions are normal, and sometimes the response is increased. If the posterior columns of the cord be diseased, as in locomotor ataxy, there is disturbance of motor power, reflex action is generally normal, and there is no degeneration of nerve or of muscle. Electrical reactions to nerve and muscle are normal. If the whole of the grey matter of the cord be diseased, as in myelitis centralis, there are paralysis, loss of reflex action, and trophic degenerative changes in nerve and muscle. The electrical reactions are the characteristic phenomena occurring with the last. There may be disease of the lateral columns combined with a lesion of the anterior horns of the grey matter, as is seen in amyotrophic lateral sclerosis. In such a case, there is motor paralysis, no loss of reflex action or degeneration of nerve, but there is marked atrophy of the muscle. Electrically, there are the usual typical reactions to the latter, the nerves remaining normal. If the posterior horns of the grey matter be diseased, as well as the posterior columns, as in some cases of multiple sclerosis or chronic myelitis, superadded to the symptoms of ataxy there are weakness of motor power and loss of reflex action, but no modification in the structure of nerve and muscle. The electrical conditions are usually normal. The disease may be situated only in the anterior horns of the grey matter, as in infantile paralysis, where it is found that the large multipolar cells are those affected. In such a case, there are motor paralysis, loss of reflex action, loss of function 
in nerve, and atrophy and degeneration of muscle. The electrical reactions are total loss of response to irritation of nerve, and typical degenerative action in muscle.

Changes are found in the anterior cornua of the grey matter in other diseases, which, however, are so different from the above as to give rise to the view that different elements must be affected. Here there is no paralysis properly so called, no loss of reflex action, no degeneration of the nerves ; but there is atrophy of the muscles. This is seen in progressive muscular atrophy, pseudo-hypertrophic paralysis, bulbar paralysis, etc. The electrical reactions are normal as regards nerve, but exhibit fully the conditions of muscular wasting.

Disease of the anterior cornua of the grey matter thus gives rise to several affections, which differ in their clinical and electrical aspects, such as amyotrophic lateral sclerosis, poliomyelitis anterior, progressive muscular atrophy, pseudo-hypertrophic paralysis, bulbar paralysis, etc. The distinctive histological differences of these have not yet been demonstrated, but the various phenomena have given rise to the probable theory that, in the anterior cornua, there are separate cellular elements presiding, some over motion, others over the nutrition of the nerve, and others over the nutrition of the muscles. As these centres are diseased either singly or combined, so are there corresponding symptoms and electrical reactions.

If the whole thickness and length of the cord be diseased, as in myelitis universalis, there is complete loss of motion, reflex action, and electrical reactions to both nerve and muscle, with atrophy of the latter, throughout the trunk and limbs.

Hitherto we have discussed the effects of destructive diseases of the cord ; but, if there be irritative lesions, such as hyperæmia, inflammation, etc., the electrical reactions to nerve and muscle are in proportion to the symptoms, as, accompanying the pain, spasm, muscular contractions, increase of reflex action, etc., their excitability is augmented to both the faradic and the galvanic stimulus.

All those cited above are more or less typical examples of definite local disorders in the spinal cord; and, although such frequently occur, still more often the conditions are much more complicated. The destructive disease may not be complete; it may invade several centres, or parts of centres; it may be complicated with irritative lesions, in which case the electrical responses may be imperfect or mixed; so much so that, in many instances, it may be almost impossible to obtain definite or reliable reactions. This, indeed, constitutes the great difficulty in the science of electro-diagnosis, which requires care, patience, and knowledge to eliminate sources of error.

Motor Nerve. - If a motor nerve be divided, or sufficiently injured or diseased, its structure undergoes marked and definite anatomical changes, which it is necessary to consider in order thoroughly to appreciate the electrical reactions which ensue; for it has been ascertained that these bear a distinct relation to the histological processes. This is typically seen in traumatic injury, the case of which we shall take as an example. During the first few days after the accident, the medullary sheath of the nerve coagulates, and gradually breaks up into longer or shorter masses, this process taking place, like all the others, near the seat of injury, and rapidly spreading to the periphery. These bodies continue to disintegrate, and, at the end of the first week, are partially converted into a mass of fine fat-globules. At this time, the degenerating fibres appear broader than before, having become infiltrated with fatty matter. At the expiration of the third or fourth week, there is a considerable diminution of the medullary masses through absorption, and also by their becoming converted into a homogeneous mass, which fills the still remaining sheath of Schwann. The fibres become slender, and ultimately remain as delicate pale bands, containing fat and increase of nuclei. In slight traumatic cases, where the nerve has not been completely divided, the axis-cylinder retains its structure till the power of conduction is re-established between the central and peripheral portions of nerve. In serious injury, where the nerve has been entirely severed, the axis-cylinder is completely destroyed in the later stages of the process of degeneration, but even then it possesses great power of resistance. Accompanying atrophy of the fibres are increased growth and hypertrophy of the neurolemma ; and, finally, true cirrhosis of the nerve is established. After a time, if there have not been complete division of the nerve, regenerative processes commence. Pale bands extend themselves from the central into the degenerated peripheral portion. These become surrounded by a medullary sheath, which slowly develops, and return of motility is observed. If the nerve have been entirely severed, the two ends are joined by fibres of new formation, commencing from the central, and afterwards proceeding from the peripheral, segment. If the nerve be only partially divided, recovery is rapid and complete. It takes place more slowly and imperfectly if it be entirely separated, according to the distance between the divided ends.

This sequence of histological changes is accompanied by definite electrical reactions. Although paralysed, the irritability of the injured nerve to both faradism and galvanism is sometimes slightly increased for the first few days ; but, after this time, there is a continuous diminution of excitability to both currents. Greater intensity is required to obtain a minimum amount of contraction. From the seventh to the twelfth day, the response to both forms of electricity disappears. This diminution commences at the portion of the nerve nearest the lesion, and very rapidly spreads to the periphery. The extinction of excitability may be lost for a variable period, and, in incurable cases, remains permanent. When regeneration in the nerve has reached a certain point, excitability to electricity recommences, the response to both currents appearing about the same period. It slowly augments, but remains a long tine below the normal standard, even after voluntary movement is appa. rently restored; indicating that, although the young nerve-fibres are capable of conveying voluntary impulses, they are not excited by the electric stimulus. The fact that the response to electricity remains long diminished after voluntary movement is completely re-established, is most probably explained by the thickening and induration of the previously diseased neurolemma acting as a bad conductor.

Besides traumatic injury, these conditions and reactions are typically seen in the so-called rheumatic paralysis of the facial nerve. Rapidly, after loss of motion has occurred, the nerve loses its response to both the faradic and the galvanic stimulus, and, in the course of about ten days, the excitability of the nerve is completely lost to both currents. The muscles soon waste, and exhibit the electrical reactions of degeneration which will subsequently be described. The process either of continued paralysis or of complete or partial recovery has already been detailed.

These electrical reactions are frequently not so typically marked as the above, indicating that the nerve-structure is not so completely disorganised. There may be only diminution, and not total loss of electric excitability, and the muscles may develop only traces of degenerative reaction, and yet there may be complete paralysis of voluntary and reflex motion, as is seen in the slight and middle forms of facial paralysis.

The same general conditions apply to all the nerves of the body if disorganised from injury or disease, and the electrical reactions vary from the slightest diminution to complete annihilation, depending entirely on the histological changes in the nerve-tissue, to which they always bear a constant relation. Not only from degeneration of the nerve itself, but, as has already been pointed out, these histological and electrical changes ensue when the centre (the spinal cord), from which the nerves take their origin, is diseased. If the nerves of the lower limbs be paralysed from affection of the brain, or of the upper portion of the cord, no abnormal electrical reactions are induced.

A portion of the nerve may be so diseased that, although it is capable of transmitting voluntary motor impulses, direct electrical applications are followed by negative results. Again, in such a case, if a healthy portion of the nerve above be stimulated, there are muscular contractions, showing that, when irritated, it is capable of originating an influence and transmitting it through the diseased part, while the latter, when directly excited, generates no function.

Voluntary Muscle. - The electrical reactions of diseased muscle differ greatly from those of diseased nerve. When the nerve supplying a muscle is injured, degeneration invariably attacks the latter, and certain anatomical changes ensue, at each stage of which there are special electrical conditions. When the functions of the nerve are thus destroyed, atrophic changes commence in the muscle during the second week, and afterwards steadily advance. In the course of five or six weeks, the fibres gradually break down, and their breadth is diminished to about one half. In slight cases, regeneration of tissue then begins to take place, mobility returns, atrophy is arrested, and the muscle slowly recovers in bulk and function. In more severe cases, in addition to the atrophy, about the second week, the transverse striæ become gradually obliterated. The fibres become cloudy in appearance, the nuclei exhibit notable multiplication in their number, chemical changes take place in the form of amyloid degeneration, and the interstitial tissue becomes infiltrated with an accumulation of cell-elements. About the sixth week, these cells become fusiform and transformed into connective tissue, which gradually increases, so that the muscle becomes dense and firm. This new structure continues to augment and undergo cicatricial contraction. This cirrhosis of the muscle, consisting of hypertrophy of the connective tissue and atrophy of the true muscular fibre, may, after a long time, be modified, and regeneration may take place, in which case new fibres gradually form, and the abnormal elements undergo degeneration, and be absorbed. Recovery only takes place after many months or years. In cases which do not recover, the destructive action continues, the hypertrophied connective tissue increases, the fibres disappear, and ultimately the muscle is converted 
into a flattened cord of simple connective tissue, which may finally be converted into fat.

The electrical reactions which accompany these anatomical changes in the muscle are definite and characteristic. If the intramuscular nerve-branches be affected, faradism applied directly acts precisely as it does on degenerated nerve ; that is, towards the end of the first week there is gradual and progressive diminution of excitability, which ends about the conclusion of the second week in complete extinction, after which the faradic current produces no effect. If the peripheral nerves remain intact, the muscles continue to contract with faradism, but slowly lose this power in proportion to the amount of muscular fibre which remains healthy, and the power is only completely lost when none of this is left.

The action of the galvanic current is quite different ; during the first week, if the nerve be also affected, like faradism, its power of producing contraction falls uniformly. In the course of the second week, there commences augmentation of galvanic excitability, which continues to increase during the following weeks. The affected muscle now contracts with currents which do not affect the normal ones. The character of the contractions is also altered, for, instead of being short and sharp, they are slow and protracted, requiring a longer application of the current to produce any result. The muscular tonus also continues cluring the whole passage of the current. Coincident with this increased excitability and different character of contraction, there are qualitative changes. This is manifested by the gradual and strong increase of the A. C. C., so that it equals or even exceeds in force the c. c. c. An opposite reaction at the opening of the current also takes place, the c. o. C. relatively increases as compared to the A. o. C., and thus the normal conditions are reversed. This sequence of events Erb has termed the "reaction of degeneration". As the muscular atrophy advances, the opening contractions disappear in the same proportion as the slowness of the contractions increases, and thcir capacity to currents of short duration diminishes. These reactions remain for from four to cight weeks, after which there is a gradual diminution of the galvanic excitability, but the qualitative changes persist. This continues, and, if the case be an incurable one with complete atrophy, all response is gradually lost, the A. C. C. being the last contraction obtainable. If regeneration and recovery take place, the normal mode of reaction is slowly re-established, galvanic excitability remaining below the normal standard.

After all these phenomena have run their coirse, and the voluntary motion and the bulk of the muscles have returned, the reaction to both the faradic and galvanic currents remains depressed; and this may continuc for a long time, due probably to the abnormal thickening of the connective tissue in nerve and muscle.

To sum up, the sequence of events, occurring in disease of nerve and muscle, in a case which ultimately recovers, is briefly as follows. Injury or disease of nerve is followed by sudden loss of voluntary motion. During the first week, there are commencing histological changes in the nerve, and no appreciable alteration in the muscle; the excitability of the nerve diminishes to both faradism and galvanism; the excitability of the muscle diminishes to faradism in the same ratio as of the nerve, and to galvanism somewhat less. During the second week, the changes in the nerve continue, and changes commence in the muscle; excitability to both currents continucs to diminish in the nerve; the muscle also exhibits continued diminution to the faradic stimulus, but to galvanism an increase of action with commencing qualitative changes. During the third week, the nerve becomes ccmpletely disorganised, and exhibits no reactions whatever to either current; the histological changes continue in the muscle whose response to faradism is lost, and to galvanism has increased, and the reaction of degeneration is fully developed. For many wecks following this condition remains, only, as atrophy advances in the muscle, the excess of galvanic excitability slowly diminishes, and the response to this with qualitative reactions is the only electrical condition to be obtained. At a varying period, from six to eight months, regencration begins to take place, and voluntary motion commences to return. Some weeks later, faradic and galvanic stimuli applied to the nerve clicit a faint response, but as yet, faradism applicd directly to the muscle is without effect, and response to galvanism is below the normal standard, although qualitative changes continue well marked. Some weeks aftcrwards, the regeneration having advanced in both nerve ancl muscle, the excitability of the nerve to both currents has incrcased, faradism now begins to incluce contractions when applied directly to the muscle, galvanism producing the same effects as before. Finally, at the end of a year or eighteen months, the nerve and muscles have regained their normal structure, with the exception of traces of the hypertrophied connective tissue, and voluntary motion has completely returned. The actions of both currents to nerve and muscle have nearly regained their quantitive and qualitative reactions, but still re- main, and continue to do so for a long time, in a state of diminished excitability.

The above is a case of moderate duration, but in some instances recovery is much more rapid, the anatomical changes being less extensive, and the electrical reactions correspondingly less marked. In others, recovery does not take place at all, when the actions to every form of the current, applied either to nerve or muscle, are ultimately annihilated.

Such cases of muscular degeneration as a result of nerve-disease arc scen clinically in traumatic injuries or in compression. They occur in affections of the nerve, as neuritis, rheumatic inflammation, tumour, etc. also from discases of the spinal cord, from which the affected nerves take their origin.

There may be profound muscular degeneration without any abnormal condition of the nerve, in which case there are no disturbed electrical changes in the latter, but a high degree of galvanic excitability and qualitative changes in the former, as is seen in progressive muscular atrophy and other discases. In these cases, there is no paralysis proper, the motor nerves give normal reactions, the muscle contracts to faradism in proportion to its bulk, and to galvanism exhibits the typical reaction of degeneration.

There is no evidence that muscle is ever diseased primarily; but its nutrition may be modificd either by disease of its nutrient centres in the cord, or by interruption of their influence by disease of the nerve. In the former case, there is no paralysis, no alnormal condition of nerve, but only degeneration of muscle; in the latter, there are paralysis, loss of excitability, and degeneration of nerve, and muscular disorganisation.

Sensory Nirves. - In health, the sensibility of different individuals to electricity varies greatly. In diseases, it may be briefly stated that, in those characterised by hypernsthesia of the sensory nerves, the sensation to both faradism and galvanism is increased in exact proportion to the extent of the affection. In the same way, when there is anzesthesia, the feeling to electricity is diminished according to the com. pleteness of that condition, and it may be entirely lost. Sometimes the sensation to faradism and galvanism may be destroyed without interference with the tactile sense, and this is obscrved in some cases of hysteria. The skin may indicate total loss of sensation, and the electromuscular sensibility remain intact.

$$
\text { [To bi concluded.] }
$$

$$
\begin{gathered}
\text { WI L L I A II BU D D, M.D., F.R.S.* } \\
\text { "IN MWMORIAM." }
\end{gathered}
$$

BY W. MCAILL CLARKE, M.R.C.S., L.S.A., Consulting Surgeon to the Bristu! (ieneral Hoipit::1; formerly Lecturer on Medical Jurisprudence at litistul Mledical School, etc.

Six and a half ycars have passed away since Dr. William Budd, smitten by severe disease, ceased to appear at our meetings; nearly seven years, during which, oblivious of the great subjects that he was accustomed to bring before us, he has suffered more than, happily, it often falls to our lot to witness, accustomed though we be to the multiform phases of disease and sickness. Even the most devoted nursing, and the must unwearying affection, were powerless to remove his suffering. Now, at last, the end has come, and the weary is at rest. During those years, it has often occurred to me -and I have no doubt the same thought has struck many other members of this Branch, that it is much to be regretted that Dr. Budd should have so finally ceised his labours amongst us without some memorial of his valuable work and genial association with us. We have had many men amongst us who have ranked high in our profession, many who have been fnmous for thcir scientific acquirements and for their general culture; but I may safely assert that of all these men, great and worthy as they have been, none will have left a richer memory behind them of successful labours in behalf of suffering humanity, of impassioned eloquence, and of great literary accomplishment, than Dr. William Budd.

On these accounts, and greatly also because of his friendship for myself, and of the great affection that I have for his memory, I am very glad, although too well aware how much better this tribute might have becn paid by many others who are now present, that his friends have put it into my power to occupy your time for a brief space this evening with an account of his life and works.

Except to our youngest members, the recollection of Dr. Budd will still be vivicl, but to very many, even already-for younger members rapidly multiply among us, older quickly disappear-his appearance and 